\title{
Urokinase-type plasminogen activator induces proliferation in breast cancer cells
}

\author{
MUKESH GANDHARI $^{1^{*}}$, NORBERT ARENS ${ }^{1 *}$, MEHER MAJETY $^{3}$, \\ ALEXANDRA DORN-BEINEKE $^{2}$ and RALF HILDENBRAND ${ }^{1}$ \\ ${ }^{1}$ Department of Pathology, ${ }^{2}$ Institute of Clinical Chemistry, University Hospital, Mannheim; \\ ${ }^{3}$ Department of Molecular Genome Analysis, German Cancer Research Center (DKFZ), Heidelberg, Germany
}

Received December 29, 2005; Accepted February 20, 2006

\begin{abstract}
Urokinase-type plasminogen activator (uPA) is implicated in various pathophysiological processes, including extracellular matrix turnover, cell migration and invasion. Our study aimed to determine the role of uPA in both proliferation and mitogen-activated protein kinase (MAPK) pathway. Hence, we analyzed the effects induced by exogeneous addition of domain-specific uPA antibodies and uPAinteracting molecules on proliferation of uPA-suppressed MDA-MB-231 breast cancer cells. uPA expression was reduced to $53 \%$ by stable transfection with an antisense/ vector construct and to $65 \%$ by siRNA transfection. Immunocytochemical Ki67 staining and flow cytometry (S-phase) analysis indicated a strong decrease of cellular proliferation activity (35\% and 38\%, respectively). Exogenous addition of high molecular weight-uPA (HMW-uPA) or incubation with the amino terminal fragment (ATF), which lacks the enzymatic activity of uPA, lead to increased cell proliferation. A strong increase of proliferation was absent when the monoclonal antiuPAR antibody IIIF10 (blocking uPA binding site), soluble uPAR (scavenger effect) and phosphatidyl-inositol-specific phopholipase C (PI-PLC, degrading uPAR) was added prior to the addition of HMW-uPA. In conclusion, HMW-uPA and ATF induce proliferation of breast cancer cells by binding to uPAR. Thereby, integrins situated adjacent to uPAR carry the signals into the cell, thus stimulating proliferation that is mediated via the MAPK pathway.
\end{abstract}

\section{Introduction}

Urokinase plasminogen activator (uPA) is a serine protease synthesized and secreted by a variety of cells in breast cancer

Correspondence to: Dr Norbert Arens, Pathologisches Institut, Universitätsklinikum Mannheim, Theodor-Kutzer-Ufer 1-3, D-68167 Mannheim, Germany

E-mail: norbert.arens@path.ma.uni-heidelberg.de

${ }^{*}$ Contributed equally

Key words: urokinase, plasminogen activator, breast cancer tissue. Binding to its GPI-anchored receptor (uPAR, CD87) on the cell surface, uPA activates plasminogen to plasmin which, in turn, degrades extracellular matrix proteins $(1,2)$. The established role of uPA in breast cancer is to proteolytically cleave the extracellular matrix (3). Furthermore, interaction of UPA to UPAR is important for adhesion, motility and migration of cells (4). It was assumed that uPA can exert cytokine-like activity by increasing the proliferation of human epidermal tumor cells and malignant renal cells $(5,6)$. uPA is subdivided into 3 domains: the $\mathrm{N}$-terminal growth factor-like domain, the kringle domain and the $\mathrm{C}$-terminal protease domain. The growth factor-like domain and kringle domain constitute the amino-terminal fragment (ATF) that binds to uPAR. The C-terminal serine protease domain activates plasminogen to plasmin, thereby inducing a cascade of events promoting the progression of cancer. The growth factor-like domain shows extensive sequence homologies to the epidermal growth factor which is assumed for acting as a mitogen (7). Plasmin is able to digest disulphide bonds in single-chain pro-uPA within the protease domain that results in two-chain active uPA (high molecular weight-uPA; HMW-uPA) (8). HMW-uPA can be further cleaved by plasmin into ATF and low-molecular-weight-uPA (LMW-uPA) that lacks the uPAR binding site. Besides its established role in proteolysis, cell adhesion, and migration, it is assumed that the uPA system is involved in cell proliferation $(7,9)$. Therefore, binding of uPA to its receptor may induce a signal transduction cascade which may lead to an increased proliferation of the cell (10). Sitrin et al demonstrated on human monocytes that UPAR is colocalized with integrins via carbohydrate-lectin interaction and that this physical association is a prerequisite for collaboration (11). Previously, we demonstrated this integrin-uPA interaction in tumor-associated macrophages of breast cancer (12). Furthermore, it was demonstrated that integrins $(\alpha 3 \beta 1)$ are associated with UPAR in MDA-MB-231 breast cancer cells (13). Since uPAR lacks transmembrane and intracytoplasmic domain for uPA-induced signal tranduction, uPAR-integrin collaboration is essential. It was demonstrated that endogenous p38 mitogen-associated protein kinase (MAPK) activity is elevated in cultured breast cancer cells and that increased p38 MAPK activity is essential for breast cancer invasiveness by stabilizing uPA mRNA (14,15). Signal transduction via integrin may induce the MAPK pathway, since recent studies have shown that p38 MAPK modulates the proliferation of cancer 
cells. Also, it was demonstrated that the proliferation of Hep3 human carcinoma cells depends on the interaction of the uPAuPAR complex with integrins, which is directly linked to the p38 MAPK pathway (Fig. 1) (16).

The aim of the present study was to analyze the mitogenic activity of uPA in breast cancer cells and to test whether ATF, the N-terminal part of the $\alpha$ chain of uPA, alone is able to transfer the growth signal and that the enzymatic domain of uPA is not relevant within this pathway. Furthermore, we investigated the pathway of uPA-induced cell proliferation by applying specific inhibitors directed against integrins and p38 MAPK.

\section{Materials and methods}

Cell culture. The human breast cancer cell line, MDA-MB231, was obtained from American Type Culture Collection (Rockville, MD, USA). The cells were routinely cultivated in Dulbecco's modified Eagle's medium (DMEM) (Gibco BRL, Invitrogen, Germany), supplemented with $10 \%$ fetal calf serum (Gibco BRL), 2 mM L-glutamine, 100 units $/ \mathrm{ml}$ penicillin, and $100 \mu \mathrm{g} / \mathrm{ml}$ streptomycin (Gibco BRL). Cells were incubated at $37^{\circ} \mathrm{C}$ in $5 \% \mathrm{CO}_{2}$. Prior to uPA stimulation experiments, cells were detached from the bottom of the culture flasks by incubation with $0.05 \%(\mathrm{w} / \mathrm{v})$ trypsin-EDTA followed by the addition of 4 volumes of DMEM/FCS. The cells were centrifuged for $5 \mathrm{~min}$ at $250 \mathrm{~g}$ and subsequently suspended in DMEM to a concentration of $10^{5}$ cells $/ \mathrm{ml}$. A cell viability test was performed with $0.4 \%$ (w/v) trypan blue reagent. For all further experiments, cell viability was always $>99 \%$.

Stable transfection and transient siRNA transfection. Stable transfection of MDA-MB-231 was performed with a pCMVdriven $u P A$-antisense vector construct (17) and an empty pCMV vector (negative control) using a transfection reagent (FuGENE 6, Roche, Mannheim, Germany). The transfection was performed with a vector:FuGENE ratio of $1: 3(\mathrm{w} / \mathrm{v})$. The cells $\left(5 \times 10^{5}\right)$ were seeded in 6-well plates on the day before transfection. The transfection complex was made with $3 \mu \mathrm{l}$ of FuGENE 6 reagent and $1 \mu \mathrm{g}$ vector DNA in serum-free medium and incubated for $15 \mathrm{~min}$ at RT. After washing the cells thoroughly with serum-free medium, the transfection complexes were transferred to the cells. The cells were incubated for $24 \mathrm{~h}$ at $37^{\circ} \mathrm{C} / 5 \% \mathrm{CO}_{2}$. Then, cells were washed twice with PBS and selective medium with geneticin (G418, $500 \mu \mathrm{g} / \mathrm{ml}$; Sigma, Munich, Germany) was added to the cells. The medium was changed every 2-4 days for 2-3 weeks to remove the debris of dead cells and to allow colonies of resistant cell clones to grow. After 3 weeks, resistant colonies were selected. Thereafter, individual colonies were propagated for further experiments.

siRNA transfection was performed as follows: on the day before transfection, cells in the exponential growth phase were harvested by trypsinization. The cells were seeded in a 6-well plate $\left(5 \times 10^{5}\right.$ cells/well). Transfection with siRNA was performed at $40-70 \%$ confluency.

Transfection was performed in a 6-well plate with FuGENE 6 reagent (Roche) according to the manufacturer's instructions [siRNA:FuGENE ratio of 1:3 (w/v)]. Sequences

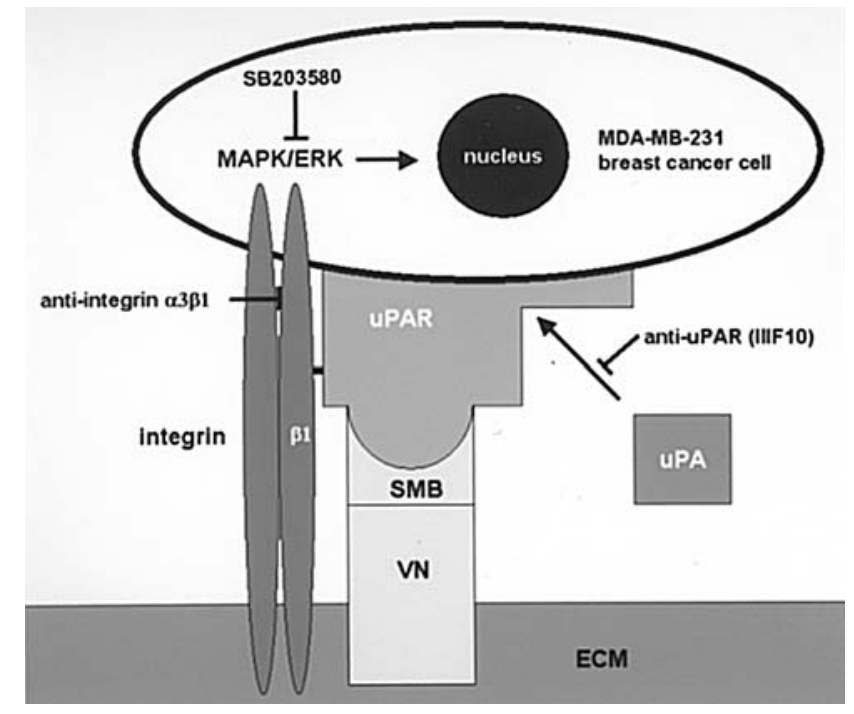

Figure 1. Proposed uPA-associated proliferation pathway in MDA-MB-231 breast cancer cells including inhibitors used in this study. Binding of uPA to UPAR induces integrin-mediated activation of the MAPK/ERK proliferation pathway. Antibody IIIF10 was applied to block uPA binding to uPAR, integrin function was blocked with anti-integrin $\alpha 3 \beta 1$ antibody, and MAPK signalling to the nucleus was blocked with the specific inhibitor, SB203580. ECM, extracellular matrix; VN, vitronectin; SMB, somatomedin B domain; MAPK, mitogen-associated protein kinase; ERK, extracellular signal-regulated protein kinase.

of $u P A$-siRNA and scrambled siRNA used in this study were described previously (17).

Each day after transfection, four of the cell culture set-ups were harvested: two set-ups (duplicate analysis) were used for a cell proliferation assay and two were used for protein extraction and Western blot analysis to analyze uPA expression.

Western blot analysis. For Western blot analysis, $10 \mu \mathrm{g}$ of total protein was separated on a sodium dodecyl sulfate (SDS)-polyacrylamide (18\%) gel and transferred (at $30 \mathrm{~mA}$ for overnight) to a nitrocellulose membrane. The membrane was incubated for $1 \mathrm{~h}$ in $5 \%$ SlimFast $^{\mathrm{TM}}$ powder in PBS. The membrane was then incubated for $1 \mathrm{~h}$ with a monoclonal antibody against uPA (American Diagnostica, Greenwich, CT, USA; 1:1000). Then, the membrane was incubated with the secondary antibody conjugated with alkaline phosphatase and finally with the chromogen, NBT/BCIP (Sigma). Protein signals on the blot were quantified by Advanced Image Data Analyzer (AIDA) software version 2.11 (Raytest, Germany) considering the non-stained region on the membrane as background (zero density units).

Immunostaining. For uPA immunofluorescence staining, the cells were seeded in four-chambered slides (Labteks, Nunc, Wiesbaden, Germany) on the day before transfection. Cells were washed thoroughly with PBS and fixed in $4 \%$ paraformaldehyde for $10 \mathrm{~min}$ prior to immunofluorescent staining for receptor-bound uPA. Cells were permeabilized with $0.1 \%$ Triton X-100 for $10 \mathrm{~min}$, washed again, and incubated in 3\% BSA (Sigma) for $1 \mathrm{~h}$. The monoclonal anti-uPA antibody (American Diagnostica) was added for $1 \mathrm{~h}$ at a 1:250 dilution. Immunoreactive sites were detected using a FITC-labeled rabbit anti-mouse IgG (1:1000, Jackson Immuno Research, 
PA, USA) for $1 \mathrm{~h}$ at room temperature and subsequently mounted in an anti-bleaching medium (Vectashield, Vector Laboratories, Burlingame, CA, USA) containing DAPI. Cells were examined using a Leica DC 500 microscope (Leica Microsystems $\mathrm{GmbH}$, Bensheim, Germany) with appropriate fluorescence filter sets.

Double immunostaining was performed using mouse monoclonal antibodies specific for human integrin B1 (CD29, 1:50; Dianova, Germany) and uPAR (IgG2a, American Diagnostica, Germany). The integrin $B 1$ antibody was visualized with a TexasRed-conjugated secondary antibody (1:1000; Jackson Immuno Research) while the biotinylated uPAR antibody (ARK biotinylation kit, Dako, Hamburg, Germany) was detected with FITC-conjugated streptavidin (1:250, Vector Laboratories). Finally, the stained cells were embedded in mounting medium as described above.

For evaluation of Ki67-associated proliferation, cells were fixed for 10 min with $4 \%$ paraformaldehyde (PFA). Cells were permeabilized with $0.1 \%$ Triton X-100 for $10 \mathrm{~min}$, washed again, and non-specific sites were blocked in $3 \%$ BSA (Sigma) for $1 \mathrm{~h}$. The slides were incubated with an anti-Ki67 antibody (Dako) for $1 \mathrm{~h}$ at 1:250 dilution at room temperature. Immunoreactive sites were detected by incubation with a 1:1000 dilution of biotin-SP-conjugated goat anti-mouse IgG/IgM (Jackson Immuno Research) for $1 \mathrm{~h}$ at room temperature and subsequent incubation with streptavidin-conjugated alkaline phosphatase (Jackson Immuno Research) diluted to 1:250 at room temperature for $30 \mathrm{~min}$. Immunoreactive sites were developed using FastRed ${ }^{\mathrm{TM}}$ substrate (Roche Diagnostics, Mannheim, Germany) containing levamisole. Slides were counterstained with hematoxylin and mounted in Kaiser's glycerol gelatin (Merck, Darmstadt, Germany). Negative controls were performed using irrelevant IgG antibodies. In each case $(n=8)$, we counted 2000 cells in various regions of the Ki67stained slides. Results are mentioned in mean percentage of Ki67-stained cells and the standard error of the mean (SEM).

FACS analysis (S-phase analysis). Trypsinized cells were fixed in ice-cold $70 \%$ ethanol for $1 \mathrm{~h}$ at room temperature. After incubation, the cells were centrifuged at $1000 \mathrm{rpm}$ for 5 min and then washed twice with PBS. The cells were resuspended in PBS containing RNase $(50 \mu \mathrm{g} / \mathrm{ml}$; Calbiochem, San Diego, CA, USA) and incubated for $30 \mathrm{~min}$ at $37^{\circ} \mathrm{C}$. Cells were then stained with propidium iodide $(50 \mu \mathrm{g} / \mathrm{ml}$; Sigma $)$ and incubated in the dark for $45 \mathrm{~min}$ at $37^{\circ} \mathrm{C}$. Fluorescence was measured using a FACScalibur ${ }^{\mathrm{TM}}$ device (BectonDickinson, USA). When bound to nucleic acids, the absorption maximum for PI is $535 \mathrm{~nm}$ and the fluorescence emission maximum is $617 \mathrm{~nm}$. PI can be excited with a xenon or mercury-arc lamp or with the 488 line of an argon-ion laser. Generally, PI fluorescence is detected in the $\mathrm{FL}_{2}$ channel of flow cytometers. A minimum of $10^{4}$ cells were measured. Data were analyzed using the CellQuestPro program (BectonDickinson) and the percentages of cells in $G_{0}, G_{1}, S$, and $G_{2} / M$ phases of the cell cycle were calculated. The total number of cells in S-phase were calculated by setting appropriate gates in the histogram. In every case, $(n=8) 10000$ cells were counted. The results are mentioned in mean percentage of S-phase fraction cells and the standard error of the mean (SEM).
Mitogenic activity of $u P A$. The mitogenic activity of uPA was determined by two experimental approaches: a) cell counting and b) cell proliferation assay by fluorogenic agent CalceinAM. Cell counting was performed in a transparent, flat-bottom, 96-well plate, whereas the proliferation assay (Ultrasensitive Cell Proliferation $\mathrm{Kit}^{\mathrm{TM}}$, Oncogene ${ }^{\mathrm{TM}}$ Research Products Inc., USA) was performed in a black, flat-bottom, 96-well plate. Stable antisense uPA-transfected cells expressing low levels of uPA were used for these experiments. Approximately 3000 cells were seeded in the 96-well plate and incubated overnight at $37^{\circ} \mathrm{C}$ and $5 \% \mathrm{CO}_{2}$. Human HMW-uPA, LMWuPA, and ATF (American Diagnostica, Greenwich, CT, USA) at appropriate concentrations were added externally to the cells. A concentration-dependent curve was generated for HMW-uPA with $0.25 \mathrm{nM}, 0.5 \mathrm{nM}, 1.0 \mathrm{nM}, 2.0 \mathrm{nM}$, and $5 \mathrm{nM}$. Stably transfected MDA-MB-231 cells were incubated with HMW-uPA, LMW-uPA, and ATF in serum-free medium for 48 h. After incubation, cells were labelled with Calcein-AM for $1 \mathrm{~h}$ at normal culture conditions. The fluorescence of the samples was measured using a fluorescence plate reader (Fluoroskan $^{\mathrm{TM}}$, Labsystems, Helsinki, Finland) (black 96-well plates) whereas, in a second set-up, cells were trypsinized and counted using a hemocytometer.

Blockage of $U P A / U P A R$ interaction and $u P A R /$ integrin interaction. Blockage of UPA/UPAR interaction was also determined by two experimental approaches as described above. Stable antisense uPA-transfected cells expressing low levels of uPA were used for experiments. Approximately 3000 cells were seeded into a 96-well plate and incubated overnight at $37^{\circ} \mathrm{C}$ in $5 \% \mathrm{CO}_{2}$. The monoclonal antibody IIIF10 $(8 \mu \mathrm{g} / \mathrm{ml}$, directed against the uPA binding region of uPAR), the monoclonal antibody IID7 $(8 \mu \mathrm{g} / \mathrm{ml}$, directed against domain II of uPAR), the soluble recombinant UPAR (3 $\mu \mathrm{g} / \mathrm{ml}$; kind gift of Professor M. Schmitt and Professor V. Magdolen, TU Munich, Germany) and bacterial PI-PLC $(5 \mu \mathrm{g} / \mathrm{ml}$; Molecular Probes, Eugene, OR, USA) in PBS were added to MDA-MB-231 uPA antisense transfectants. Two hours later, HMW-uPA incubation followed by repeated addition of the same amount every $24 \mathrm{~h}$ for $48 \mathrm{~h}$. After incubation, the cells were labelled with Calcein-AM for $1 \mathrm{~h}$ at normal culture conditions. The fluorescence of the samples was measured using a fluorescence plate reader (Fluoroskan ${ }^{\mathrm{TM}}$ ). Whereas, for cell counting, cells were trypsinized and counted using a hemocytometer. It was demonstrated by Wei et al that a3ß1 integrins are associated with UPAR in MDA-MB-231 breast cancer cells (13). uPAR-dependent functions are blocked by antibodies to $\alpha 3$ (P1B5). These antibodies are reported to inhibit $\alpha 3 \beta 1$ function, but they do not block the association of uPAR and $\alpha 3$. For blocking the uPAR-integrin binding, the MDA-MB-231 cells were incubated for $6 \mathrm{~h}$ at $37^{\circ} \mathrm{C}$ with anti-integrin $\alpha 3$ (anti-CD 49c) clone P1B5 (5 $\mu \mathrm{g} / \mathrm{ml}$ ) (\# MAB 1952Z, Chemicon, Germany). The proliferation rate was determined as mentioned above. Negative controls were performed using irrelevant IgG antibodies.

MAP kinase assay. MAP kinase assay was also performed by applying two experimental approaches as described above. Stable antisense uPA-transfected cells cultured for $24 \mathrm{~h}$ in $10 \%$ FCS/DMEM were washed twice with serum-free medium. 


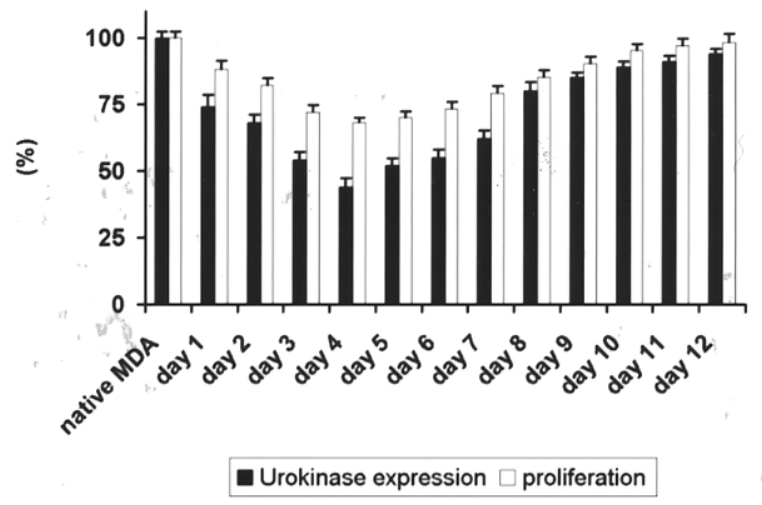

Figure 2. Simultaneous presentation of the uPA protein level (Western blot analysis) and proliferation activity (proliferation assay) of siRNA-transfected MDA-MB-231 breast cancer cells; data are given in mean percentage \pm SEM (compared to native MDA-MB-231 cells). The maximum uPA suppression of siRNA-transfected MDA-MB-231 cells was achieved after 4 days $(24.14 \pm 0.62$ d.u., $\mathrm{p}<0.001 ; \mathrm{n}=8)$; this time point of maximum $\mathrm{uPA}$ inhibition coincides with the time point of maximum proliferation decrease $(68 \pm 1.8 \%$, $\mathrm{p}<0.001 ; \mathrm{n}=8)$.

The effect of SB203580 (Calbiochem) inhibition on p38 MAPK activation was tested by treating the cells with $10 \mu \mathrm{M}$ SB203580 for $48 \mathrm{~h}$ in serum-free DMEM. The experiment was performed in three set-ups: in the first set-up the cells were cultivated in medium without FCS and with HMW-uPA for $24 \mathrm{~h}$, the second set-up was treated with SB203580 $(10 \mu \mathrm{M})$ for $48 \mathrm{~h}$ and the final set-up was treated with a combination of HMW-uPA $(1 \mathrm{nM})$ and SB203580 $(10 \mu \mathrm{M})$ for $48 \mathrm{~h}$. The proliferation rate was measured using the Ultra Sensitive Proliferation $\mathrm{Kit}^{\mathrm{TM}}$ and by cell counting.

Statistical analysis. Statistical analyses were performed to determine the significance of changes in UPA expression and proliferation status. We compared the native MDA-MB-231 cells with transfected cells by performing Student's t-test, Scheffe's test, and analysis of variance (ANOVA). Student's t-test was used to assess between-group differences in mean values for each sample in proliferation assay for siRNA transfectant cells, whereas Scheffe's test was performed for all the remaining experiments.

\section{Results}

Stable transfection of MDA-MB-231 cells with an antisenseuPA-construct induced a decrease in uPA expression by $53 \%$. A stronger effect was observed after transfection of uPA siRNA molecules: this procedure led to a transient suppression of uPA expression with a maximum of $65 \%$ at day 4 after transfection (Fig. 2). Densitometric data from the Western blots revealed that the stably antisense-uPA-transfected MDA-MB231 cells showed significantly $(\mathrm{p}<0.001)$ lower uPA expression [30.87 \pm 1.91 density units (d.u.)] when compared to native (65.04 \pm 1.49 d.u.) and vector control transfected $(62.12 \pm 2.18$ d.u.) cells.

Densitometric data of Western blot analysis of siRNAtransfected cells revealed a continuous decrease of uPA expression from $55.48 \pm 2.1 \mathrm{~d}$.u at day 1 after transfection to a minimum expression of $25.84 \pm 0.62$ d.u. at day 4 after transfection. Re-increase was observed with $94 \%$ of initial
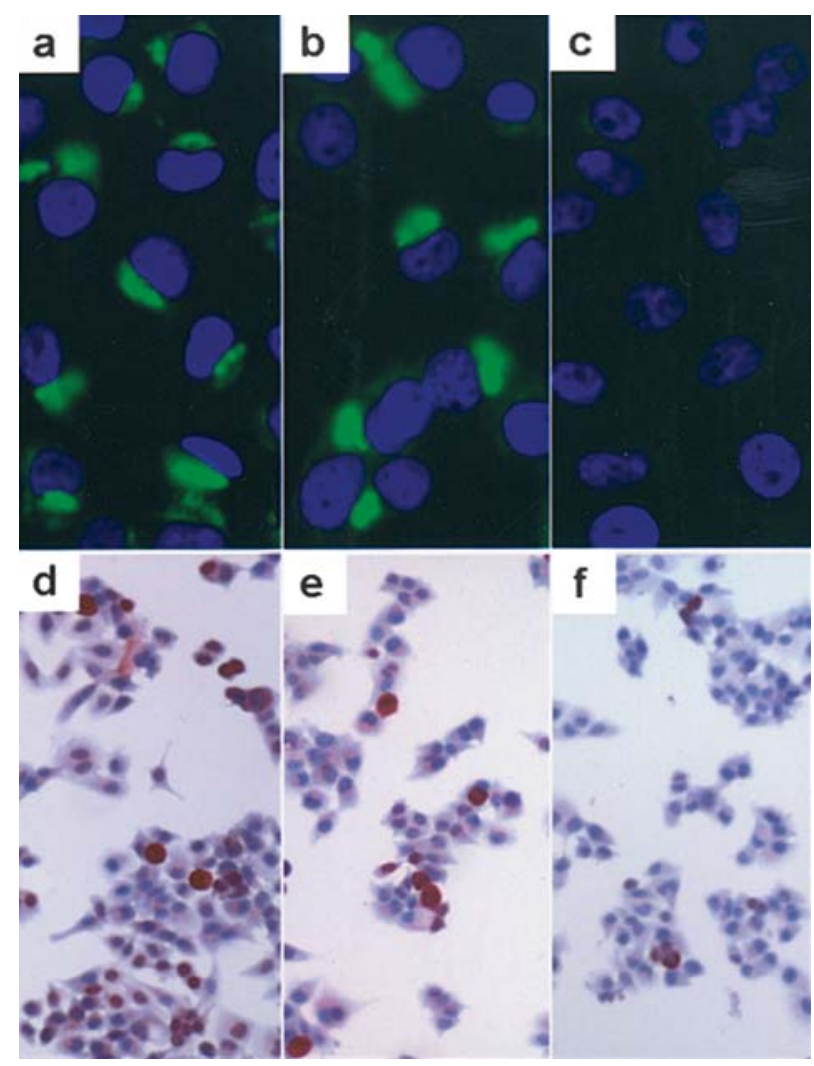

Figure 3. (a-c) uPA immunofluorscence staining of MDA-MB-231 cells (uPA, green; nuclei, DAPI-stained blue). (a and b) Strong positive anti-uPA staining in native MDA-MB-231 cells (a) and pCMV-transfected MDA-MB-231 cells (control) (b); (c) in stably antisense-transfected MDA-MB-231 cells, only a faint anti-uPA immunoreaction is seen. (d-f) Ki67 immunostaining of MDAMB-231 cells (nuclei: red). (d and e) High Ki67-associated proliferation rate in native MDA-MB-231 cell (d) and pCMV-transfected MDA-MB-231 cells (control) (e); (f) low Ki67-associated proliferation rate in stably antisensetransfected MDA-MB-231 cells.

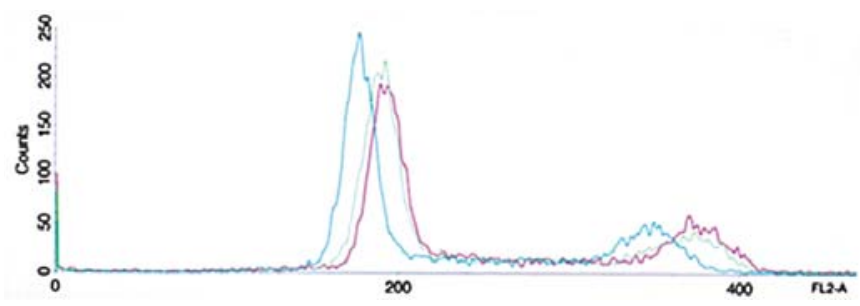

Figure 4. Flow cytometry analysis (FACS) (a) native MDA-MB-231 cells (red) (b) pCMV-transfected MDA-MB-231 cells (control) (green) and (c) stably antisense-transfected MDA-MB-231 (blue). $10^{4}$ cells of each celltype were analyzed. The mean percentage of cells in S-phase was $100 \pm 2.2 \%$ in native MDA-MB-231 cells and 97 $\pm 1.2 \%$ in pCMV-transfected MDAMB-231 cells (control) whereas, in stably antisense-transfected MDA-MB231 cells, we observed a significantly reduced S-phase fraction of $62 \pm 1.2 \%$ $(\mathrm{p}<0.001, \mathrm{n}=8)$.

expression at day 12 after transfection. The uPA expression in siRNA-transfected MDA-MB-231 cells was significantly different $(\mathrm{p}<0.001)$ to that of native MDA-MB-231 cells (74.97 \pm 2.17 d.u.) and scrambled siRNA-transfected MDAMB-231 cells (70.56 \pm 0.42 d.u.) (control).

Data from proliferation assay revealed that uPA-suppressed cells were significantly less proliferating in both antisensevector-transfected and siRNA-transfected cells as compared 


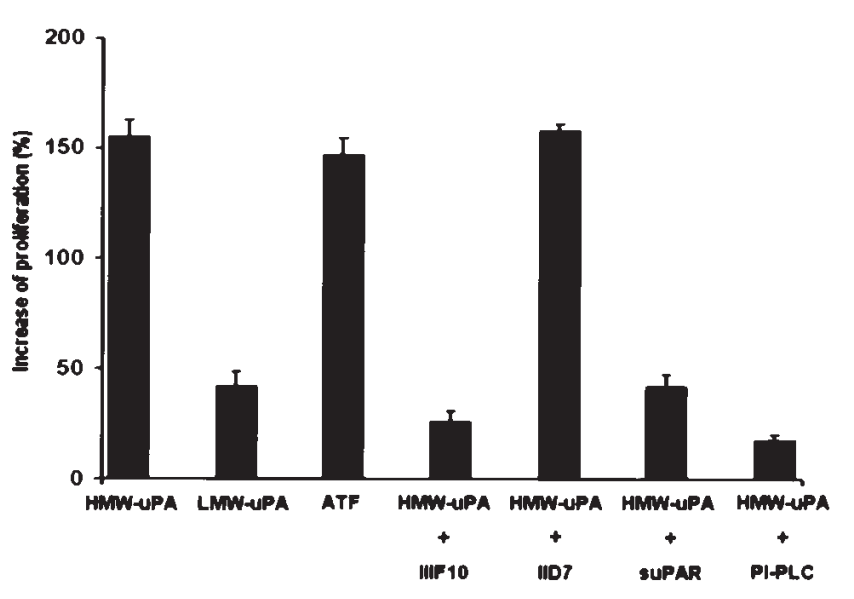

Figure 5. Mitogenic effect of uPA on stably transfected MDA-MB-231 cells. Cells were incubated with high molecular weight uPA (HMW-uPA), low molecular weight uPA (LMW-uPA) and amino-terminal fragment (ATF). After $48 \mathrm{~h}$, the proliferation rate determined by proliferation assay was compared with proliferation of transfected cells which have not received $\mathrm{uPA}$ or ATF. Increase of proliferation (y-axis) is demonstrated as mean \pm SEM. After incubation of cells with LMW-uPA, the increase in proliferation was significantly lower than that of cells which were incubated with HMW-uPA $(\mathrm{p}<0.001 ; \mathrm{n}=8)$. After incubation of cells with ATF, the increase in proliferation was not significantly different compared to cells which were incubated with HMW-uPA. To block the uPA binding site of uPAR, cells were incubated with mAb IIIF10 prior to the addition of HMWuPA. In this experiment, the increase in proliferation was significantly lower than that of cells which were incubated with mAb IID7 (binding to domain II of uPAR) and HMW-uPA. After incubation with soluble uPAR and HMWuPA, cell proliferation was also significantly reduced (scavanger effect). When cells were treated with phosphoinositol-specific phospholipase C (PI-PLC) prior to the addition of HMW-uPA, the increase in proliferation was significantly reduced.

with cells transfected with scrambled siRNA (control). In siRNA-transfected cells, the proliferation rate measured by the proliferation assay and cell counting decreased during the first 4 days. From day 4 after transfection, the proliferation of the cells re-increased continuously (Fig. 2).

The data from Western blotting and proliferation assay showed that the proliferation activity is strongly linked to the level of uPA expression.

The antisense uPA-transfected cells were tested for uPA expression by immunofluorescence and immunocytochemistry. The monoclonal anti-uPA antibody was utilized for staining the cells. Only a faint fluorescence signal was observed in antisense uPA-transfected cells by immunofluorescence and immunocytochemistry. In contrast, the MDA-MB-231 and vector-transfected MDA-MB-231 cells (control) showed high expression levels of uPA (Fig. 3a-c).

To investigate the expression of the proliferation marker Ki67 in breast cancer cells, we performed immunocytochemical staining. The monoclonal Ki67 antibody binds strongly to the nuclei of MDA-MB-231 cells. The proliferation rate of native MDA-MB-231 cells and empty-pCMVtransfected MDA-MB-231 cells (control) was 46.5 $\pm 2.1 \%$ and $45.1 \pm 1.4 \%$ respectively. Antisense uPA-transfected cells revealed a significantly reduced Ki67-related proliferation rate of $30.5 \pm 1.3 \%(p<0.001)$ (Fig. 3d-f). These results represent an absolute Ki67-prolifer-ation reduction of $16 \%$ and a relative Ki67-proliferation reduction of $35 \%$ in stable antisensetransfected MDA-MB-231 cells.
Flow cytometry analysis. S-phase flow cytometry analysis was performed to determine the proliferation status of the cells. The histogram of the cells transfected with antisense uPA vector revealed decreased levels of propidium iodide fluorescence intensity as compared to untransfected and vector-transfected cells (control) (Fig. 4). The percentage of cells in S-phase were significantly reduced in antisense uPAtransfected cells compared to the controls $(\mathrm{p}<0.001)$. In total, 10000 MDA-MB-231 cells were analyzed. The percentage of cells in S-phase was $14.38 \pm 2.2 \%$ for native MDA-MB-231 cells and $13.97 \pm 1.2 \%$ for pCMV (control)-transfected MDAMB-231 cells whereas, in antisense uPA-transfected cells, we found an S-phase fraction of $9.03 \pm 1.6 \%$. These results represent an absolute reduction of S-phase fraction of 5.35\% and relative reduction of S-phase fraction of $38 \%$ in stably antisense-transfected MDA-MB-231 cells.

Role of uPA in breast cancer cell proliferation. To investigate a possible role of different domains of UPA as mitogenic activator we exogenously added HMW-uPA, LMW-uPA and ATF to the antisense uPA transfectants and detected their proliferation activity by both fluorogenic assay and cell counting. By addition of HMW-uPA to antisense uPA transfectants, we observed an increased fluorescence-associated cell proliferation in a concentration-dependent manner (the following values represent the mean \pm SEM fluorescence intensity: $0.25 \mathrm{nM}, 12.65 \pm 1.1 ; 0.5 \mathrm{nM}, 13.63 \pm 2.9 ; 1 \mathrm{nM}$, $18.42 \pm 1.4 ; 2 \mathrm{nM}, 14.98 \pm 1.2 ; 5 \mathrm{nM}, 13.33 \pm 1.2)$. Maximum proliferation was observed at a uPA concentration of $1 \mathrm{nM}$ HMW-uPA within $48 \mathrm{~h}$ as compared to untreated cells. For further experiments, we used a concentration of $1 \mathrm{nM} \mathrm{HMW-}$ uPA. Human HMW-uPA and ATF were found to stimulate cell proliferation determined by both fluorogenic assay (Fig. 5) and cell counting in breast cancer cells. The fluorescenceassociated proliferation for incubation of antisense-transfected cells with HMW-uPA and ATF was $19.84 \pm 0.76$ and $17.85 \pm 1.5$ respectively. These results represent an increase of proliferation of $155 \pm 7.7 \%$ and $147 \pm 7.4 \%$. In contrast, the addition of LMWuPA showed no significant increase $(10.05 \pm 1.2 ; 42 \pm 8.6 \%)$ in proliferation $(\mathrm{p}<0.001)$.

Inhibition of proliferation by blocking $U P A / u P A R$ interaction. The monoclonal antibody IIIF10 directed against the uPA binding region of UPAR was added to MDA-MB-231 antisense transfectants $2 \mathrm{~h}$ prior to uPA stimulation followed by repeated additions of the same amount of mAb IIIF10 every $24 \mathrm{~h}$. However, after $48 \mathrm{~h}, \mathrm{mAb}$ IIIF10 had significantly suppressed the uPA-mediated mitogenic activity. The monoclonal antibody, mAb IID7, directed against domain II of uPAR (mAb IID7 does not block the uPA - uPAR binding) produced no termination of the UPA-mediated mitogenic activity. The addition of recombinant soluble UPAR resulted in an abrogation of the stimulation induced by HMW-uPA, thereby leading to a significantly suppressed mitogenic cellular response $(\mathrm{p}<0.001)$.

The effect of removal of the GPI-anchored uPA receptor from the cell surface was also tested. It was shown by Fischer et al that treatment of ovarian cancer cell line OV-MZ-6 antisense transfectants with PI-PLC $(5 \mu \mathrm{g} / \mathrm{ml})$ released $80 \%$ of the cell surface uPA receptors (7). Our results from breast 


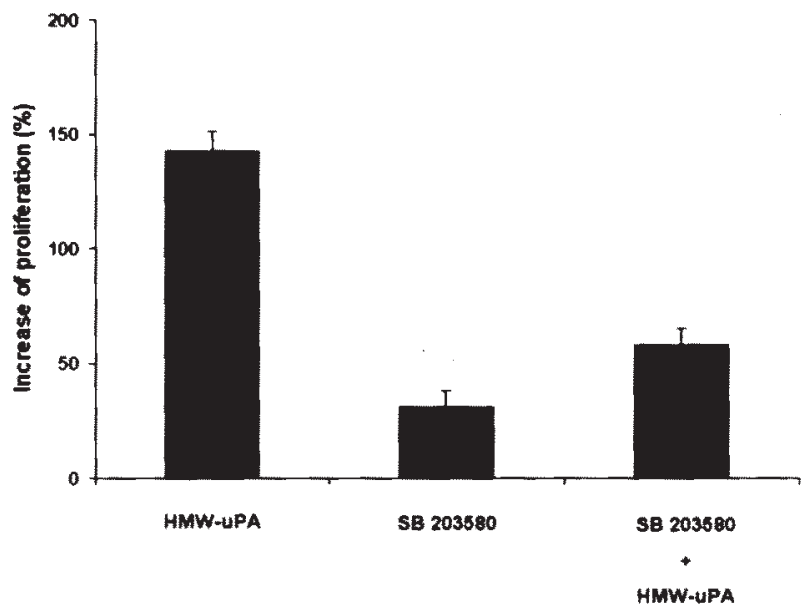

Figure 6. Effect of p38 MAPK inhibition on proliferation of stable transfectant MDA-MB-231 cells. The stable transfected MDA-MB-231 cells were treated with high molecular weight-uPA (HMW-uPA) and SB203580 $(10 \mu \mathrm{M})$ separately and together respectively. Cell proliferation was determined by cell proliferation assay within $48 \mathrm{~h}$. Increase of proliferation (y-axis) is demonstrated as mean \pm SEM. To block the p38 MAPK pathway, cells were incubated with SB203580 prior to the addition of HMW-uPA. Increase of proliferation is significantly lower compared to cells which were incubated with HMW-uPA alone $(\mathrm{p}<0.001 ; \mathrm{n}=8)$.

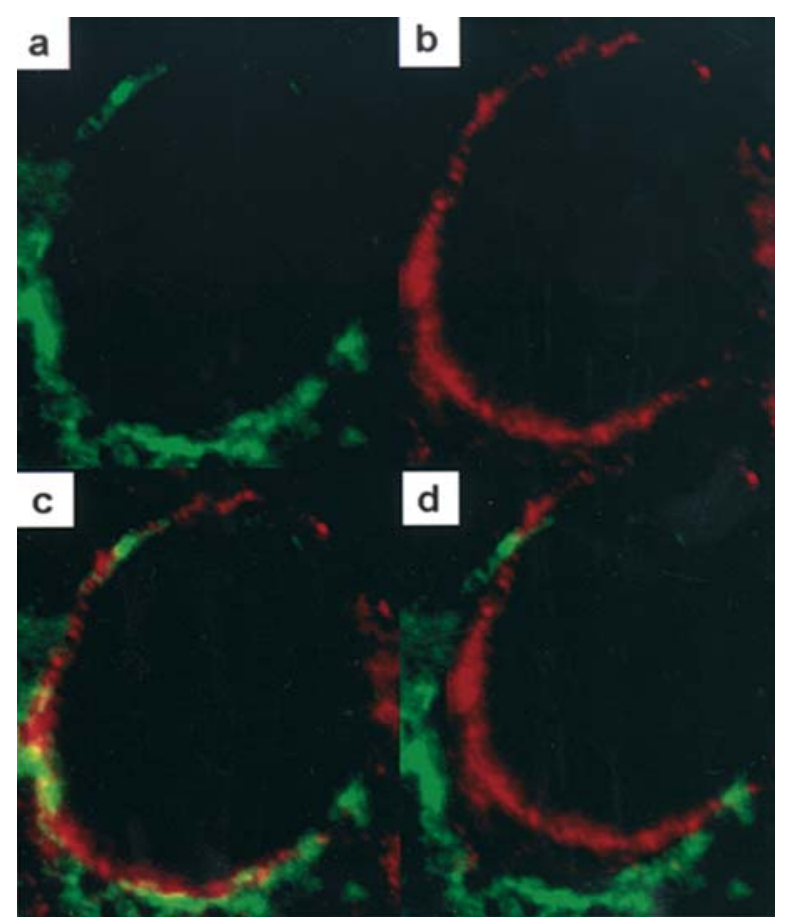

Figure 7. Double immunofluorescence staining of MDA-MB-231 cells with antibodies specific for UPAR (a) and integrin B1 (b); both signals are colocalized near the cell membrane (c). A manually performed pixel shift of the red and green staining strongly supports the highly similar staining results for $\mathrm{UPAR}$ and integrin $B 1$ (d).

cancer cell line MDA-MB-231 are in agreement with the findings in OV-MZ-6 cells: absence of uPAR on the PI-PLCtreated antisense-transfected cells showed nearly no mitogenic activity (Fig. 5). However, when cells were stimulated with HMW-uPA, the decrease in cell number in antisense transfectants was observed, presumably due to reduction of
uPAR on the cell surface. The mean values ( \pm SEM) obtained in fluorogenic assay by incubation of antisense-transfected cells with IIIF10, IID7, suPAR and PI-PLC prior to HMWuPA were $11.73 \pm 0.92(26 \pm 4.6 \%), 18.38 \pm 1.01(158 \pm 3.0 \%)$, $12.98 \pm 1.15(42 \pm 5.2 \%)$ and $7.99 \pm 0.41(18 \pm 2.1 \%)$ respectively.

To block the uPAR-integrin function in MDA-MB-231 cells, they were treated with a monoclonal anti- $\alpha 3 \beta 1$ antibody prior to addition of HMW-uPA. For negative control, an irrelevant IgG antibody was used (data not shown). The fluorescence intensities determined by a fluorogenic proliferation assay were as follows: monoclonal anti- $\alpha 3 \beta 1$ (alone), 9.34 \pm 1.6 ; HMW-uPA (alone), 20.14 \pm 1.9 ; monoclonal anti$\alpha 3 ß 1+$ HMW-uPA, $10.58 \pm 1.1(\mathrm{p}<0.001)$.

Association of 338 MAP kinase activity with breast cancer cell proliferation. The p38 MAPK signaling pathway has been shown to mediate the expression of uPA induced by various extracellular stimuli. We hypothesized that constitutive p38 MAPK signaling might be important for uPA-induced tumor cell proliferation. Several related pyridinyl imidazole compounds have been found to be highly specific inhibitors of p38 MAPK. SB203580, a member of this group, is a highly selective inhibitor of p38 MAPK, which does not alter the activity of other MAPKs including ERKs and JNKs. We thus used this inhibitor to investigate the role of p38 MAPK activity in proliferation of MDA-MB-231 breast cancer cells. Antisense-transfected MDA-MB-231 cells were treated with SB203580 $(10 \mu \mathrm{M})$ prior to addition of HMW-uPA. This treatment revealed a significant inhibition of cellular proliferation detected by cell counting and fluorogenic assay (Fig. 6) $(\mathrm{p}<0.001)$. The fluorescence-associated proliferation for incubation of antisense-transfected cells with SB203580, SB203580 addition prior to HMW-uPA and HMW-uPA detected by fluorogenic assay were $8.20 \pm 0.4(31 \pm 1.5 \%)$, $10.67 \pm 1.6(58 \pm 3.7 \%)$ and $19.09 \pm 0.83(143 \pm 3.1 \%)$ respectively.

\section{Discussion}

Previously we have determined a strong correlation between uPA levels in tumor tissue extracts and Ki67-associated proliferation rate in breast cancer (18). This prompted us to investigate the mitogenic activity of uPA in the breast cancer cell line, MDA-MB-231, in detail.

To determine the proliferation rate in breast cancer cells, we have established a uPA-suppressed model by two approaches, they include transfection of an antisense-vector construct and siRNA transfection of MDA-MB-231 breast cancer cells (17). Our results indicate that suppression of uPA $(65 \%)$ by siRNA transfection is associated with a strong decrease of proliferation rate with maximum at the 4th and the 5th day after transfection (38\%) (Fig. 2). From 6 days after transfection, the suppression effect lessened, and normal uPA levels were detected 12 days after transfection. In accordance with this increase in uPA expression from the 6th day, the proliferation rate of MDA-MB-231 cells increased in the same way (Fig. 2). The fact that the siRNA effect starts fading from the 6th day after transfection was also previously described by other authors (19). The loss of siRNA effect or propagative system over a longer period of time is due to an absence of the RNA-dependent RNA polymerase homologue 
in humans. The propagative system was well demonstrated in C. elegans and Drosophila (19). Using conventional transfection methods, we obtained a stable and permanent uPA-suppression $(53 \%)$, which was also associated with a strong decrease in cell proliferation (32\%) (Fig. 2).

In order to characterize the mitogenic activity of uPA in breast cancer cells, we examined the S-phase fraction of the antisense uPA transfectants by flow cytometry analysis. As described above, the suppression of UPA in breast cancer cells decreases cell proliferation. The DNA histograms of S-phase fraction confirmed that antisense uPA-transfected cells revealed significantly reduced DNA synthesis as compared to controls (38\%) (Fig. 4). These findings indicate that uPA is involved in proliferation of breast cancer cells.

To confirm the required presence of uPA in the proliferation of breast cancer cells, we performed experiments with antisense uPA transfectants treated with HMW-uPA, LMWuPA and ATF. The mitogenic activity of HMW-uPA on antisense uPA-transfected cells showed a concentrationdependent effect with maximum growth stimulation at $1 \mathrm{nM}$. With increasing uPA concentrations, the proliferation rate decreased again. These findings are consistent with earlier reports concerning uPA antisense transfectants of human ovarian cancer cell line OV-MZ-6 (7).

Treatment of distinct components of uPA to MDA-MB231 cells have confirmed that HMW-uPA and ATF were most effective in inducing cell proliferation, whereas nearly no stimulation was observed when cells were incubated with LMW-uPA. This is not surprising since LMW-uPA lacks the uPAR binding site (growth factor-like domain). These data indicate that the growth factor-like domain in HMW-uPA and ATF is required for uPA-induced cell proliferation. Similarly, in ovarian cancer cells, non-glycosylated recombinant ATF showed mitogenic activity (7). In contrast, two authors reported no mitogenic activity of ATF in human smooth muscle cells and the breast cancer cell line BC $8701(20,21)$.

To prove that uPA-induced proliferation is mediated via uPAR, we performed blocking experiments using two antiUPAR antibodies named IIIF10 and IID7. The antibody, IIIF10, binds to domain III and blocks uPAR binding, whereas IID7 binds to domain II without blocking the uPA-uPAR interaction. The proliferation rate is significantly decreased when cells are incubated with monoclonal antibody IIIF10 prior to the addition of HMW-uPA. However, this effect was not observed when cells were incubated with monoclonal antibody IID7 prior to incubation with HMW-uPA (Fig. 5). Kirchheimer and coworkers have found similar results in human melanoma cell line GUBSB using monoclonal anti-uPA antibody (MPW5UK) which blocks binding of uPA to uPAR (22).

Additionally, we have treated transfected MDA-MB-231 cells with PI-PLC to degrade uPAR on the cell surface. PI-PCL removes several GPI-anchored proteins (among them uPAR) from the cell surface so that the effects observed might also be influenced by the removal of other GPI-anchored proteins.

Consecutive incubation of the PI-PLC-treated cells with HMW-uPA revealed only a slight increase in cell proliferation (Fig. 5). This little proliferation effect of HMW-uPA might be due to few uPAR molecules still remaining on the cell surface. When HMW-uPA was added, with soluble uPAR which lacks the GPI anchor, the proliferation rate was strongly reduced. This effect is due to the soluble uPAR acting as scavanger for uPA, despite the fact that affinity of suPAR to uPA is reduced approximately 1500 times as compared with cell-bound uPAR. In this way, the level of free HMW-uPA in supernatant is reduced and proliferation activity is inhibited.

The results of these experiments revealed that the uPAUPAR interaction is necessary for uPA-induced proliferation. We have demonstrated by immunohistochemistry that integrin and UPAR are co-localized in native MDA-MB-231 cells (Fig. 7). Since uPAR lacks transmembrane and intracytoplasmic domains for uPA-induced signal transduction, uPAR-integrin interaction is necessary. To test this assumption, we used a monoclonal anti- $\alpha 3 \beta 1$ antibody (P1B5) to block uPAR-integrin function in MDA-MB-231 cells. The proliferation rate was significantly reduced when this antibody was added prior to the addition of HMW-uPA. Considering our results, we hypothesized that the mitogenic activity of uPA is mediated via integrins adjacent to uPAR. It was reported by Sitrin et al that integrins and uPAR are associated via carbohydrate-lectin interaction and that this physical association is necessary for collaboration (23). Recent studies have shown that integrins can activate the p38 MAPK pathway and that $\alpha_{v}$ integrin, p38 MAPK and uPA are functionally linked in breast cancer cells (14). These earlier observations prompted us to investigate whether the p38 MAPK pathway is important for breast cancer cell proliferation. The treatment of invasive breast cancer MDA-MB-231 cells with p38 MAPK inhibitor SB203580 prior to the addition of HMW-uPA significantly abrogates the proliferation of these cells (Fig. 6), thus improving the importance of the p38 MAPK signaling pathway in the proliferation of breast cancer cells.

\section{Acknowledgements}

This research was supported by the Deutsche Krebshilfe, Dr Mildred-Scheel-Stiftung für Krebsforschung, Germany, $10-1507 \mathrm{H}$, and by research funds of the Faculty of Clinical Medicine Mannheim, University of Heidelberg, Germany. Printing of this manuscript was supported by kind gifts from American Diagnostica GmbH, Pfungstadt, Germany and the Lesser-Loewe-Foundation e.V., Mannheim, Germany)

\section{References}

1. Zamarron C, Lijnen HR, van Hoef B and Collen D: Biological and thrombolytic properties of proenzyme and active forms of human urokinase-I. Fibrinolytic and fibrinogenolytic properties in human plasma in vitro of urokinases obtained from human urine or by recombinant DNA technology. Thromb Haemost 52: $19-23,1984$

2. Gurewich V, Pannell R, Louie S, Kelley P, Suddith RL and Greenlee R: Effective and fibrin-specific clot lysis by a zymogen precursor form of urokinase (pro-urokinase). A study in vitro and in two animal species. J Clin Invest 73: 1731-1739, 1984.

3. Holst-Hansen C, Johannessen B, Hoyer-Hansen G, Romer J, Ellis V and Brunner N: Urokinase-type plasminogen activation in three human breast cancer cell lines correlates with their in vitro invasiveness. Clin Exp Metastasis 14: 297-307, 1996.

4. Lu H, Mabilat C, Yeh P, Guitton JD, Li H, Pouchelet M, Shoevaert D, Legrand Y, Soria J and Soria C: Blockage of urokinase receptor reduces in vitro the motility and the deformability of endothelial cells. FEBS Lett 380: 21-24, 1996.

5. Kirchheimer JC, Wojta J, Christ G, Hienert G and Binder BR: Mitogenic effect of urokinase on malignant and unaffected adjacent human renal cells. Carcinogenesis 9: 2121-2123, 1988 . 
6. Kirchheimer JC, Wojta J, Christ G and Binder BR: Proliferation of a human epidermal tumor cell line stimulated by urokinase. FASEB J 1: 125-128, 1987.

7. Fischer K, Lutz V, Wilhelm O, Schmitt M, Graeff H, Heiss P, Nishiguchi T, Harbeck N, Kessler H, Luther T, Magdolen V and Reuning U: Urokinase induces proliferation of human ovarian cancer cells: characterization of structural elements required for growth factor function. FEBS Lett 438: 101-105, 1998.

8. Kasai SH, Arimura A, Nishida M and Suyama T: Proteolytic cleavage of single-chain pro-urokinase induces conformational change which follows activation of the zymogen and reduction of its high affinity for fibrin. J Biol Chem 260: 12377-12381, 1985.

9. Rabbani SA, Mazar AP, Bernier SM, Haq M, Bolivar I, Henkin J and Goltzman D: Structural requirements for the growth factor activity of the amino-terminal domain of urokinase. J Biol Chem 267: 14151-14156, 1992.

10. Seddighzadeh M, Zhou JN, Kronenwett U, Shoshan MC, Auer G, Sten-Linder M, Wiman B and Linder S: ERK signalling in metastatic human MDA-MB-231 breast carcinoma cells is adapted to obtain high urokinase expression and rapid cell proliferation. Clin Exp Metastasis 17: 649-654, 1999.

11. Sitrin RG, Todd RF, Albrecht E and Gyetko MR: The urokinase receptor (CD87) facilitates CD11b/CD18-mediated adhesion of human monocytes. J Clin Invest 97: 1942-1951, 1996.

12. Hildenbrand R, Wolf G, Böhme B, Bleyl U and Steinborn A: Urokinase plasminogen activator receptor (CD87) expression of tumor-associated macrophages in ductal carcinoma in situ, breast cancer, and resident macrophages of normal breast tissue. J Leukoc Biol 66: 40-49, 1999.

13. Wei Y, Eble JA, Wang Z, Kreidberg JA and Chapman HA: Urokinase receptors promote betal integrin function through interactions with integrin alpha3beta1. Mol Biol Cell 12: 2975-2986, 2001.

14. Chen J, Baskerville C, Han Q, Pan ZK and Huang S: Alpha(v) integrin, p38 mitogen-activated protein kinase, and urokinase plasminogen activator are functionally linked in invasive breast cancer cells. J Biol Chem 276: 47901-47905, 2001.
15. Huang S, New L, Pan Z, Han J and Nemerow GR: Urokinase plasminogen activator/urokinase-specific surface receptor expression and matrix invasion by breast cancer cells requires constitutive p38alpha mitogen-activated protein kinase activity. J Biol Chem 275: 12266-12272, 2000.

16. Aguirre Ghiso JA, Kovalski K and Ossowski L: Tumor dormancy induced by downregulation of urokinase receptor in human carcinoma involves integrin and MAPK signaling. J Cell Biol 147: 89-104, 1999.

17. Arens N, Gandhari M, Bleyl U and Hildenbrand R: In vitro suppression of urokinase plasminogen activator in breast cancer cells - a comparison of two antisense strategies. Int J Oncol 26: 113-119, 2005.

18. Hildenbrand R, Dilger I, Horlin A and Stutte HJ: Urokinase and macrophages in tumor angiogenesis. Br J Cancer 72: 818-823, 1995.

19. Holen T, Amarzguioui M, Wiiger MT, Babaie E and Prydz H: Positional effects of short interfering RNAs targeting the human coagulation trigger tissue factor. Nucleic Acids Res 30: 1757-1766, 2002.

20. Kanse SM, Benzakour O, Kanthou C, Kost C, Lijnen HR and Preissner KT: Induction of vascular SMC proliferation by urokinase indicates a novel mechanism of action in vasoproliferative disorders. Arterioscler Thromb Vasc Biol 17: 2848-2854, 1997.

21. Luparello $\mathrm{C}$ and Del Rosso M: In vitro anti-proliferative and anti-invasive role of amino-terminal fragment of urokinase-type plasminogen activator on $8701-\mathrm{BC}$ breast cancer cells. Eur J Cancer 32A: 702-707, 1996.

22. Kirchheimer JC, Wojta J, Christ G and Binder BR: Functional inhibition of endogenously produced urokinase decreases cell proliferation in a human melanoma cell line. Proc Natl Acad Sci USA 86: 5424-5428, 1989.

23. Sitrin RG, Pan PM, Blackwood RA, Huang J and Petty HR: Cutting edge: evidence for a signaling partnership between urokinase receptors (CD87) and L-selectin (CD62L) in human polymorphonuclear neutrophils. J Immunol 166: 4822-4825, 2001. 